\title{
EZH2 inhibitor GSK343 inhibits sepsis-induced intestinal disorders
}

\author{
DONGYOU YUE ${ }^{1}$, ZHIYING WANG ${ }^{2}$, YONGAN YANG ${ }^{1}$, ZHENJUN HU $^{1}$, GANPING LUO $^{1}$ and FU WANG FU $^{3}$ \\ Departments of ${ }^{1}$ Emergency and ${ }^{2}$ Neurology, The First People's Hospital of Chenzhou City, Chenzhou, Hunan 423000; \\ ${ }^{3}$ Department of Critical Care Medicine, Zhangye Second People's Hospital, Zhangye, Gansu 734000, P.R. China
}

Received August 6, 2020; Accepted December 4, 2020

DOI: $10.3892 /$ etm.2021.9854

\begin{abstract}
Enhancer of zeste homolog 2 (EZH2) is positively associated with poor clinical outcomes in a number of aggressive tumors. Recent studies have demonstrated that inhibition of EZH2 also suppressed the inflammatory response during sepsis. The present study aimed to investigate whether an inhibitor of EZH2, GSK343, could protect the intestine against sepsis-induced injury in vivo. Mice underwent cecal ligation and perforation (CLP) to induce sepsis and were assigned into three groups: Sham, CLP and CLP + GSK343. For GSK343 treatment, the septic mice were intravenously injected with GSK343 at $6 \mathrm{~h}$ post-CLP. The results indicated that EZH2 was highly expressed while tight junction (TJ) proteins ZO-1, occludin and claudin-1 expression was reduced in the intestinal tissue of mice subjected to CLP compared with the sham group. CLP operation also caused intestinal pathological injury and the production of inflammatory cytokines including TNF- $\alpha$, IL-1 $\beta$ and IL- 6 in both serum and intestinal tissues. Meanwhile, CLP induced cell apoptosis of intestinal tissue based on the increased number of apoptotic cells, reduced expression of Bcl-2 and higher expression of caspase- 3 and Bax. However, the presence of GSK 343 partially rescued intestinal pathological injury, reduced the level of inflammatory cytokines, repressed cell apoptosis and promoted TJ protein expression. Finally, the decreased number of Paneth cells caused by CLP operation was reversed by GSK343 treatment. In conclusion, the results of the present study demonstrated that GSK343 could protect the intestine against sepsis-induced injury in vivo. Inhibition of EZH2 may provide a therapeutic approach for intestinal dysfunction during sepsis.
\end{abstract}

Correspondence to: Dr Fu Wang, Department of Critical Care Medicine, Zhangye Second People's Hospital, Linsong West Street, West Third Ring, Zhangye, Gansu 734000, P.R. China E-mail:wangfuun@126.com

Key words: enhancer of zeste homolog 2, intestine, inflammation, sepsis

\section{Introduction}

Sepsis is an infection-induced systemic inflammatory response syndrome that is aggressive, can rapidly progress to septic shock or multiple organ dysfunction syndrome (MODS), is difficult to treat and has a poor prognosis (1). Therefore, early identification, timely diagnosis and effective treatment of sepsis may be the key to preventing and treating MODS and improving the survival rate of patients with sepsis (2). The current treatment measures for sepsis are mainly symptomatic treatment, anti-infection agents and organ support treatment (3). Despite the continuous development and advancement of clinical treatment measures, the pathogenesis of sepsis is complicated, is closely associated with the pathophysiological changes of multiple organs and contributes to a high mortality rate (4). Therefore, an in-depth exploration of the pathogenesis of sepsis, active prevention of the occurrence and improvement of the cure rate of patients with sepsis are important issues that need to be resolved urgently in clinical practice and have important practical significance.

As an important tissue and organ of the human body, the intestine is not only the main place for the digestion, absorption and transportation of nutrients, but also an important immune protection site for the body (5). Recent studies have indicated that the intestine serves an important role in the pathophysiological development of critical illnesses with its unique physiological environment (6). In addition to causing an uncontrolled systemic inflammatory response, sepsis often leads to the malfunction of a variety of organs of the body, whereby the intestine is one of the most sensitive organs (7). During sepsis, bacteria and toxins in the intestinal lumen can activate intestinal immune cells, releasing a large number of inflammatory mediators, such as TNF- $\alpha$, IL-1 $\beta$ and IL-6, and causing intestinal mucosal damage (8). Simultaneously, inflammatory mediators interact and form a network to cause mediator cascade effects, leading to a vicious cycle, accelerating the course of the disease, and even causing MODS (9). As a result, the intestinal mucosa not only functions as a barrier but can also produce a variety of inflammatory mediators after being attacked (8). It is currently proposed that intestinal function may not only be impaired due to the development of sepsis, but also cause excessive release of inflammatory mediators through the translocation of bacteria and toxins, leading to dysfunction and even failure of other important organs (10). 
Therefore, suppressing the inflammatory response in the intestine is crucial for preventing the initiation and progression of sepsis and MODS.

Enhancer of zeste homolog 2 (EZH2) is an important member of the polycomb-group protein gene family. Histone methyltransferase homologous sequence 2 can catalyze the trimethylation of amino acid K27 at position 27 of histone $\mathrm{H} 3$ $(\mathrm{H} 3 \mathrm{~K} 27 \mathrm{Me} 3)$ and regulate cell proliferation (11). In addition, the gene expression product of EZH2 can silence tumor suppressor genes and serve an important role in the evolution of various tumors. EZH2 is found to be positively associated with poor clinical outcomes in a number of aggressive tumors, and its inhibition has been indicated to effectively inhibit cell proliferation and prevent tumor progression $(12,13)$. A previous study demonstrated that inhibition of EZH2 suppressed the progression of lung injury and alleviated inflammation induced by sepsis, suggesting that EZH2 could be a potential biomarker in predicting clinical outcome and a novel target for therapeutic interference in sepsis (14). However, the role of $\mathrm{EZH} 2$ in intestinal disorders caused by sepsis remains to be elucidated. The present study aimed to investigate whether an inhibitor of EZH2, GSK343, could protect the intestine against sepsis-induced injury in mice that underwent cecal ligation and perforation (CLP) operation.

\section{Materials and methods}

Animals. A total of 30 C57BL/6 mice (17-20 g) were purchased from the Chinese Academy of Medical Sciences. All mice were kept in a specific pathogen-free environment at room temperature under a controlled 12/12 h light/dark cycle and received food and water ad libitum. Male mice at 6-8 weeks of age were used for the experiments. The animal study was approved by the Institutional Animal Care and Use Committee of the Second People's Hospital of Zhangye City.

Establishment of a sepsis model. A sepsis model was established by performing CLP on mice. All mice were anesthetized with $2 \%$ isoflurane inhalation for about 3 min until they were totally anesthetized before undergoing surgical procedures, and their body temperatures were maintained at $36-38^{\circ} \mathrm{C}$ with a heating pad. During the surgical procedure, $1.5 \%$ isoflurane was used to maintain anesthesia. A $1.5 \mathrm{~cm}$ incision was made on the abdominal wall, and the cecum was exposed and ligated $0.5 \mathrm{~cm}$ from the tip with a $4-0$ silk suture. The cecum was perforated by a single puncture with a 21-gauge needle and gently squeezed to extrude a small amount of feces from the perforation site. The cecum was replaced into the abdominal cavity, and the exposed abdominal wall was closed in two layers with a running 4-0 silk suture. The sham-operated group $(n=10)$ only underwent laparotomy. Mice were resuscitated with subcutaneous injection of $1 \mathrm{ml}$ normal saline.

Animal grouping and treatment. At $6 \mathrm{~h}$ after the CLP operation, sepsis could be induced and the successfully modeled mice were selected and randomly subdivided into CLP $(n=10)$ and CLP + GSK343 ( $n=10)$ groups. For GSK343 treatment, the septic mice were intravenously injected with GSK343 (0.2 ml/20 g; MedChemExpress) suspended in anhydrous ethanol diluted in PBS at $6 \mathrm{~h}$ post-CLP. For the CLP group, mice were intravenously injected with equivalent amounts of anhydrous ethanol. At $72 \mathrm{~h}$ after CLP or sham operation, all mice were euthanized by cervical dislocation and small intestine samples and serum were collected.

Histological staining. The small intestine samples were fixed with $4 \%$ paraformaldehyde overnight at $4^{\circ} \mathrm{C}$, then dehydrated through an alcohol-xylene series and finally embedded in paraffin and stored at room temperature. Sections $(3 \mu \mathrm{m}$ thickness) were cut, deparaffinized, rehydrated with gradient ethanol and stained with hematoxylin and eosin (H\&E; Abcam) or TUNEL. H\&E staining allows the identification of Paneth cells located at the base of intestine crypt based on their distinctive granule staining (15). TUNEL staining was used to stain apoptotic cells according to the manufacturer's instructions (Beyotime Institute of Biotechnology).

For immunofluorescent staining forZO-1, deparaffinized and rehydrated sections were blocked with $10 \%$ goat serum (Gibco; Thermo Fisher Scientific, Inc.) at $37^{\circ} \mathrm{C}$ for $1 \mathrm{~h}$ and incubated with a primary antibody against ZO-1 (Abcam; cat. no. ab190085; $1: 2,000)$ at $4^{\circ} \mathrm{C}$ overnight. Following incubation with IgG-FITC secondary antibody (cat. no. ab6881; 1:5,000; Abcam) at room temperature for $1 \mathrm{~h}$ in the dark, samples were stained with DAPI at room temperature for $20 \mathrm{~min}$ in the dark and photographed under a fluorescence microscope.

Western blot analysis. Intestinal tissues were pulverized in liquid nitrogen and lysed in ice-cold RIPA buffer (Beyotime Institute of Biotechnology) containing $0.01 \%$ protease and phosphatase inhibitor (Sigma-Aldrich; Merck KGaA). Protein concentration in the supernatant was measured using a BCA protein assay. Equal amounts of protein $(20 \mu \mathrm{g} /$ lane $)$ were subjected to $10 \%$ SDS-PAGE and transferred to PVDF membranes. The transferred proteins were blocked with $5 \%$ non-fat milk for $2 \mathrm{~h}$ at room temperature. Following blocking, the membranes were incubated with anti-EZH2 (cat. no. 5246; 1:1,000), ZO-1 (cat. no. 13663; 1:1,000), occludin (cat. no. 91131; 1:1,000), claudin-1 (cat. no. 13255; 1:1,000), TNF- $\alpha$ (cat. no. 11948; 1:1,000), IL-1 $\beta$ (cat. no. 31202; 1:1,000), IL-6 (cat. no. 12912; 1:1,000), and anti-GAPDH (cat. no. 5174; 1:1,000) primary antibodies (all, Cell Signaling Technology, Inc.) overnight at $4{ }^{\circ} \mathrm{C}$ and then incubated with respective HRP-conjugated secondary antibodies (anti-rabbit IgG; cat. no. 7074; 1:3,000; Cell Signaling Technology, Inc.). Protein signals were visualized using the super ECL detection reagent (Beyotime Institute of Biotechnology). Image-Pro Plus software version 6.0 (Roper Technologies, Inc.) was used for densitometry.

ELISA. Serum supernatant was separated after centrifugation and the levels of TNF- $\alpha$ (cat. no. ab208348), IL-1 $\beta$ (cat. no. ab197742) and IL-6 (cat. no. ab100713) (all from Abcam) were tested using ELISA test kits in accordance with the manufacturer's instructions. The optical density value was measured at an excitation wavelength of $450 \mathrm{~nm}$ using a microplate reader (Bio-Rad Laboratories, Inc.) with the blank well serving as the control.

$R N A$ extraction and reverse transcription-quantitative PCR $(R T-q P C R)$. Total RNA of the intestinal tissues was extracted 

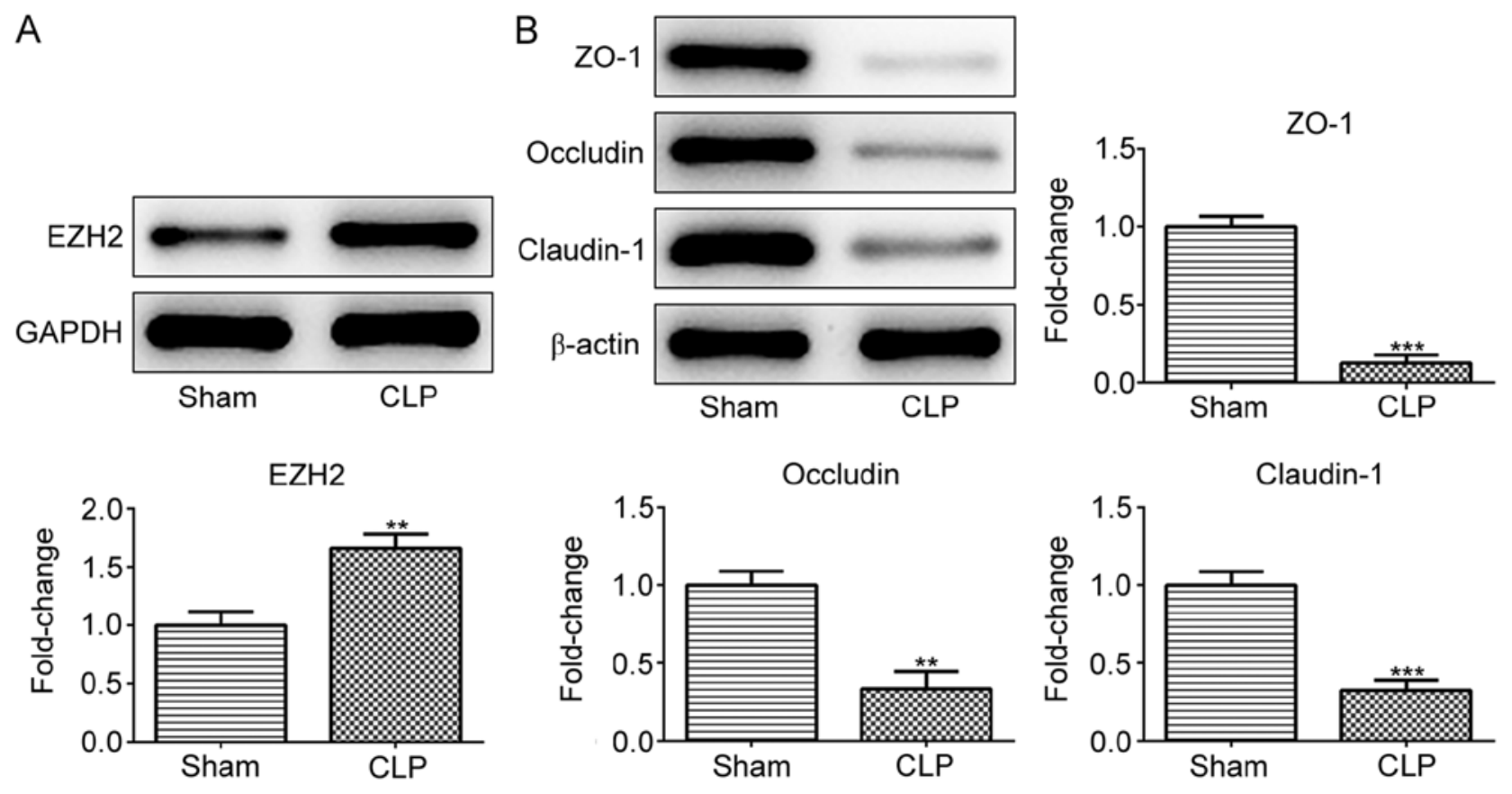

Figure 1. EZH2 is upregulated while tight junction proteins are downregulated upon CLP stimulation. The protein expression of (A) EZH2 and (B) tight junction proteins including ZO-1, occludin and claudin-1 in the intestinal tissues of Sham and CLP mice $(\mathrm{n}=10) .{ }^{* * *} \mathrm{P}<0.01$ and ${ }^{* * * *} \mathrm{P}<0.001 \mathrm{vs}$. Sham. EZH2, enhancer of zeste homolog 2; CLP, cecal ligation and perforation.

using TRIzol ${ }^{\circledR}$ reagent (Invitrogen; Thermo Fisher Scientific, Inc.). RT of the extracted RNA was completed with a two-step method according to the kit instructions (SuperScript ${ }^{\mathrm{TM}}$ III Two-Step RT-PCR system; Thermo Fisher Scientific, Inc.). A TaqMan probe (Takara Bio, Inc.) was used for qPCR. The following primer pairs were used for qPCR analysis: ZO-1 forward, 5'-GGAGCAGGCTTTGGAGGAG-3' and reverse, 5'-TGGGACAAAAGTCCGGGAAG-3'; occludin forward, 5'-GTGAATGGGTCACCGAGGG-3' and reverse, 5'-AGA TAAGCGAACCTGCCGAG-3'; claudin-1 forward, 5'-GGC TTCTCTGGGATGGATCG-3' and reverse, 5'-GCAGCA GTT-CACAGGCAAAA-3' and GAPDH forward, 5'-GGT CCCAGCTTAGGTTCATCA-3' and reverse, 5'-ATCCGTTCA CACCGACCTTC-3'. The following thermocycling conditions were used for the qPCR: Initial denaturation at $95^{\circ} \mathrm{C}$ for $30 \mathrm{sec}$; and 40 cycles of denaturation at $95^{\circ} \mathrm{C}$ for $10 \mathrm{sec}$, annealing at $60^{\circ} \mathrm{C}$ for $20 \mathrm{sec}$ and extension at $70^{\circ} \mathrm{C}$ for $10 \mathrm{sec}$. GAPDH was used as the internal reference gene and the $2^{-\Delta \Delta \mathrm{Cq}}$ method (16) was utilized to analyze the expression of the target genes.

Statistical analysis. All experiments were performed at least three times. Data were expressed as the mean \pm SD and plotted and analyzed using GraphPad Prism 5 (GraphPad Software, Inc.). Differences between groups were analyzed using one-way ANOVA followed by Tukey's post hoc test. $\mathrm{P}<0.05$ was considered to indicate a statistically significant difference.

\section{Results}

EZH2 is upregulated while tight junction (TJ) proteins are downregulated in the intestinal tissue of a CLP mice model. To determine whether EZH2 serves a role in sepsis-induced intestinal disorders, EZH2 expression was detected in CLPor sham-operated mice. As presented in Fig. 1A, EZH2 expression was significantly higher in the intestinal tissues of the CLP group compared with the sham group, indicating the potential role of EZH2 in sepsis-induced intestinal disorders. Subsequently, the expression of TJ proteins including ZO-1, occludin and claudin-1 was measured in the intestinal tissues of sham- or CLP-operated mice. The results revealed that ZO-1, occludin and claudin-1 protein expression was reduced upon CLP stimulation (Fig. 1B).

GSK343 inhibits CLP-induced intestinal pathological injury and inflammation. GSK343, which is an EZH2 inhibitor, was used to treat CLP mice to observe whether inhibition of EZH2 could protect the intestine against sepsis-induced injury. At the end of the experiment, animals were sacrificed and $\mathrm{H} \& \mathrm{E}$ staining was performed on small intestinal tissue sections. Fig. 2A demonstrates the results of $\mathrm{H} \& \mathrm{E}$ staining in the intestinal tissues of mice in different groups. The ileum tissue structure of the mice in the sham operation group was normal, the intestinal villi were arranged neatly and the villi structure was clear. In the CLP group, however, evident edema, hyperemia, necrosis and inflammatory cell infiltration were observed, accompanied by missing apical epithelial cells of the villi and thinner and shorter microvilli. Compared with the CLP group, the intestinal structure of the mice in the GSK343 treatment group was relatively normal, with relieved intestinal villi edema and inflammation. The levels of inflammatory cytokines, including TNF- $\alpha$, IL-1 $\beta$ and IL- 6 , in the serum and intestinal tissues of mice in different groups are presented in Fig. 2B and C. It was indicated that CLP stimulation significantly promoted the production of all inflammatory cytokines, and this increase was reversed by GSK343 treatment.

GSK343 represses CLP-induced intestinal tissue apoptosis. Subsequently, the cell apoptosis of animal intestinal tissue in 
A
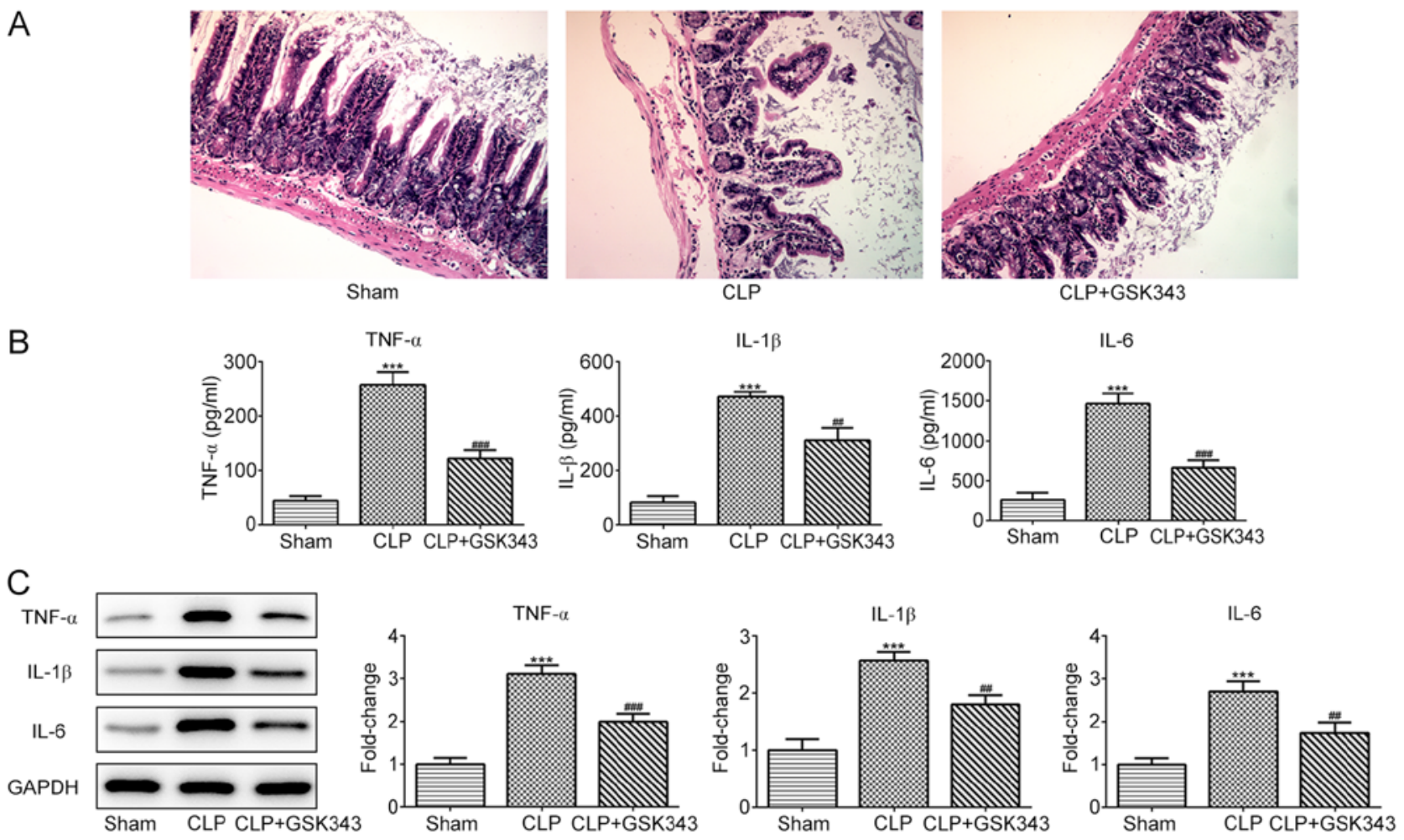

Figure 2. Effects of GSK343 on CLP-induced morphological changes and inflammation in intestinal tissues. (A) Representative hematoxylin and eosin images for intestinal tissues of mice in the Sham, CLP and CLP + GSK343 groups (magnification, $x 200$ ). (B) The concentration of TNF- $\alpha$, IL-1 $\beta$ and IL-6 in the serum of mice in the Sham, CLP and CLP + GSK343 group ( $\mathrm{n}=10)$. (C) The expression of TNF- $\alpha$, IL-1 $\beta$ and IL-6 in the intestinal tissues of mice in the Sham, CLP and CLP + GSK343 group $(\mathrm{n}=10) .{ }^{* * * *} \mathrm{P}<0.001$ vs. Sham; ${ }^{\# t} \mathrm{P}<0.01$ and ${ }^{\# \# \#} \mathrm{P}<0.001$ vs. CLP. CLP, cecal ligation and perforation.

different groups was assessed. As presented in Fig. 3A, CLP operation markedly increased the number of apoptotic cells compared with the sham operation. However, in contrast to the CLP group, GSK343 treatment markedly reduced the number of apoptotic cells in the intestinal tissue of CLP mice. Similar results were observed in Fig. 3B and C, where CLP reduced anti-apoptotic protein $\mathrm{Bcl}-2$ expression and increased the expression of pro-apoptotic proteins Bax and cleaved-caspase-3. Meanwhile, GSK343 treatment attenuated the effects of CLP on apoptotic protein expression.

GSK343 promotes TJ protein expression and the number of Paneth cells in the intestinal tissue of a CLP mice model. The expression of TJ proteins including ZO-1, occludin and claudin-1 in the intestinal tissue of mice was also detected. The results indicated in Fig. 4 revealed that mRNA and protein expression of these $\mathrm{TJ}$ proteins were significantly downregulated upon CLP stimulation, but effectively partially recovered by GSK343 treatment. Consistently, as presented in Fig. 5, ZO-1 immunofluorescent staining results showed the decreased expression of ZO-1 upon CLP stimulation, but effectively recovered expression of ZO-1 after GSK343 treatment.

Finally, the distribution of Paneth cells at the base of the intestine crypt was observed based on their distinctive granule staining. As presented in Fig. 6, CLP mice showed a significant decrease in the number of crypts with positive Paneth cells in the small intestine compared with Sham mice. By contrast, animals treated with GSK343 exhibited an increased number of Paneth cells compared with CLP mice.

\section{Discussion}

Intestinal mucosal destruction, intestinal wall congestion and necrosis caused by sepsis are the main causes of intestinal dysfunction (17). In the present study, a mice sepsis model was constructed by CLP operation. Following which, the mucosal morphology was markedly damaged, the expression of TJ proteins was decreased, in conjunction with the production of a large amount of inflammatory cytokines and occurrence of apoptosis. TJ proteins serve an important role in the physiology and disease biology of intestinal disease (18). TJ proteins exert their functional role as integral proteins in forming barriers in the gut (19). However, TJ proteins can also serve important functional roles in the signaling, trafficking and regulation of gene expression (20). In addition, the number of Paneth cells were significantly decreased upon CLP stimulation. Paneth cells are physiologically found at the bottom of small intestinal crypts and are characterized by their apically located granules (21). The intestinal crypts have been identified as a significant source of antimicrobial peptides and proteins that are important in host defense and in shaping the composition of the commensal microbiota (22). The dysfunction of Paneth cells and molecular mechanisms underlying the secretory disorders of Paneth cells have been demonstrated to be highly associated with inflammatory bowel disease (23). Consistent with previous reports $(22,24,25)$, the present results demonstrated sepsis-induced intestinal injury and the involvement of Paneth cell dysfunction.

EZH2 serves crucial roles in regulating a variety of cellular functions, including development and differentiation (26). 

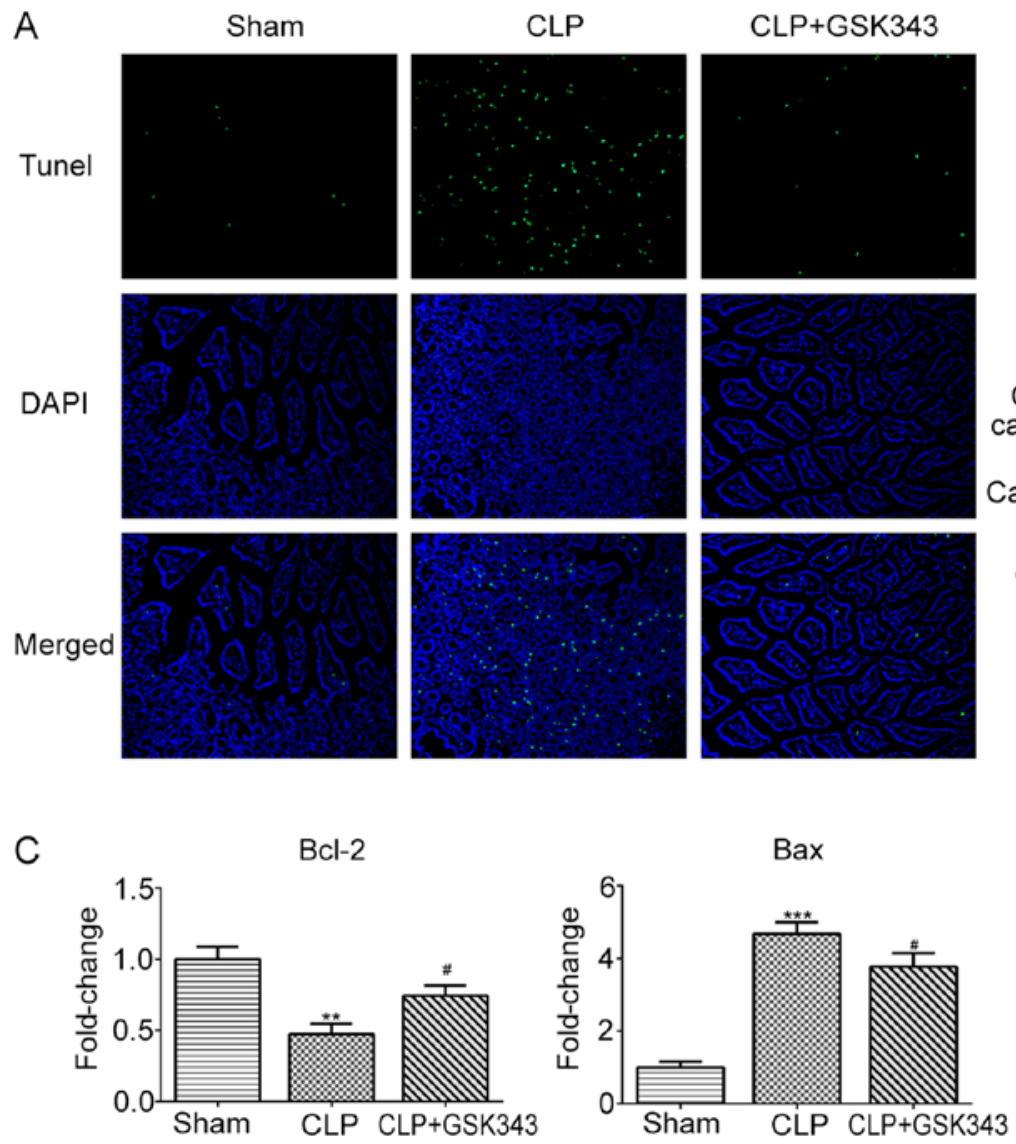

B
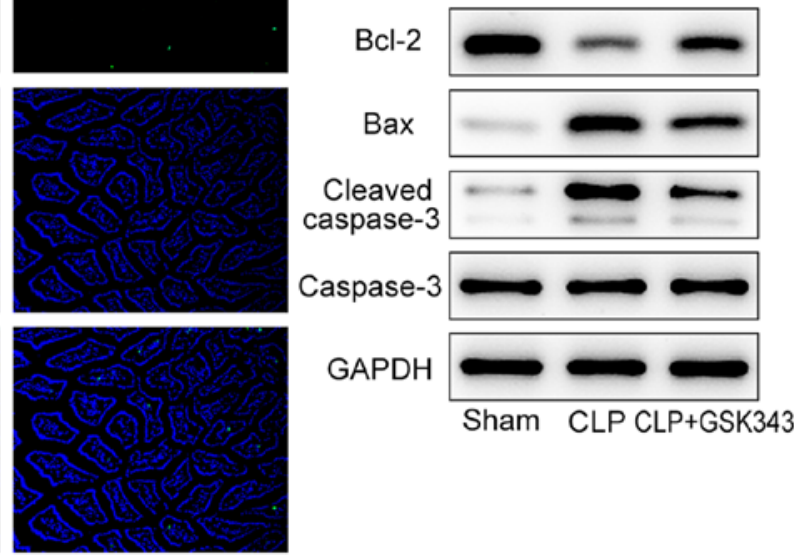

Cleaved caspase-3/Caspase-3

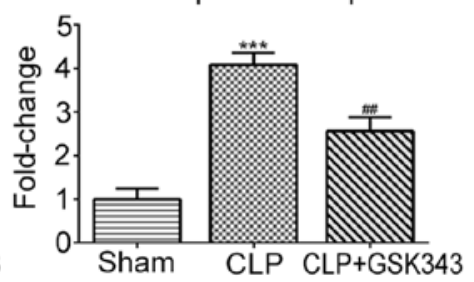

Figure 3. Effects of GSK343 on CLP-induced apoptosis in intestinal tissues. (A) Representative TUNEL staining images of intestinal tissues of mice in different groups (magnification, x200). Apoptotic cells are stained green. DAPI staining represents the nuclei. (B and C) The expression of apoptosis-related proteins including Bcl-2, Bax and cleaved-caspase-3 in the intestinal tissues of mice in the Sham, CLP and CLP + GSK343 group ( $\mathrm{n}=10){ }^{* *} \mathrm{P}<0.01$ and ${ }^{* * * *} \mathrm{P}<0.001$ vs. Sham; ${ }^{*} \mathrm{P}<0.05$ and ${ }^{\# \#} \mathrm{P}<0.01$ vs. CLP. CLP, cecal ligation and perforation.

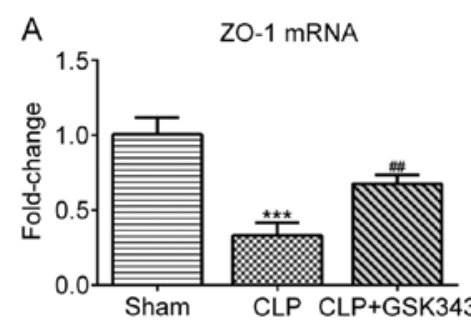

B
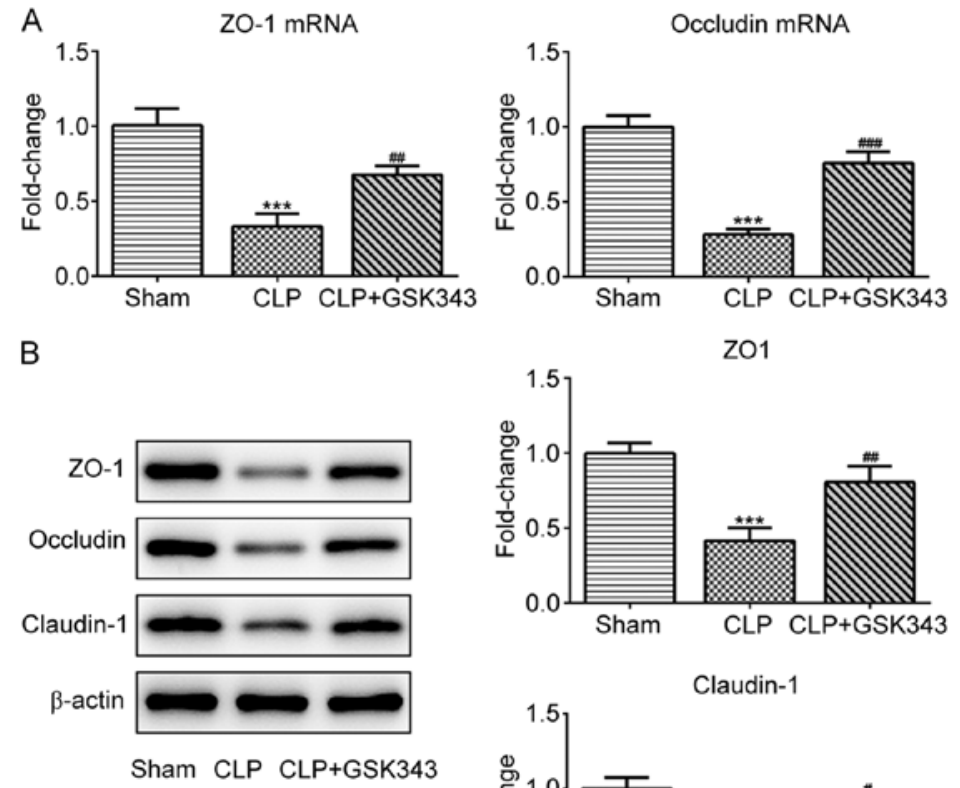

ZO1
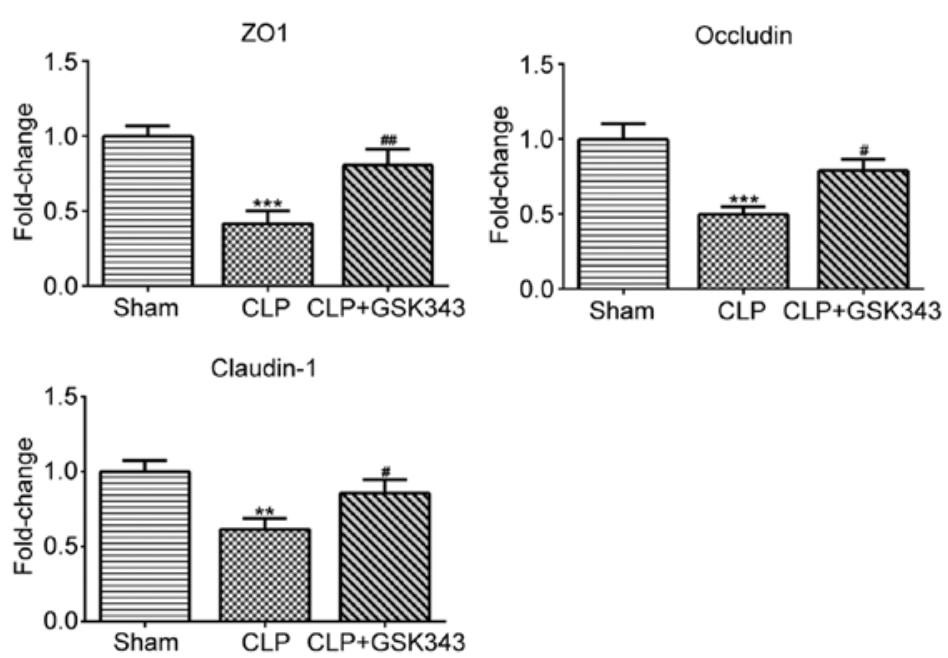

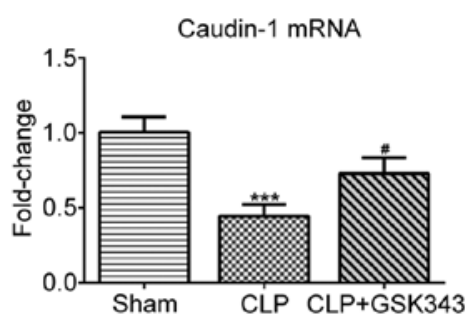

(a) 


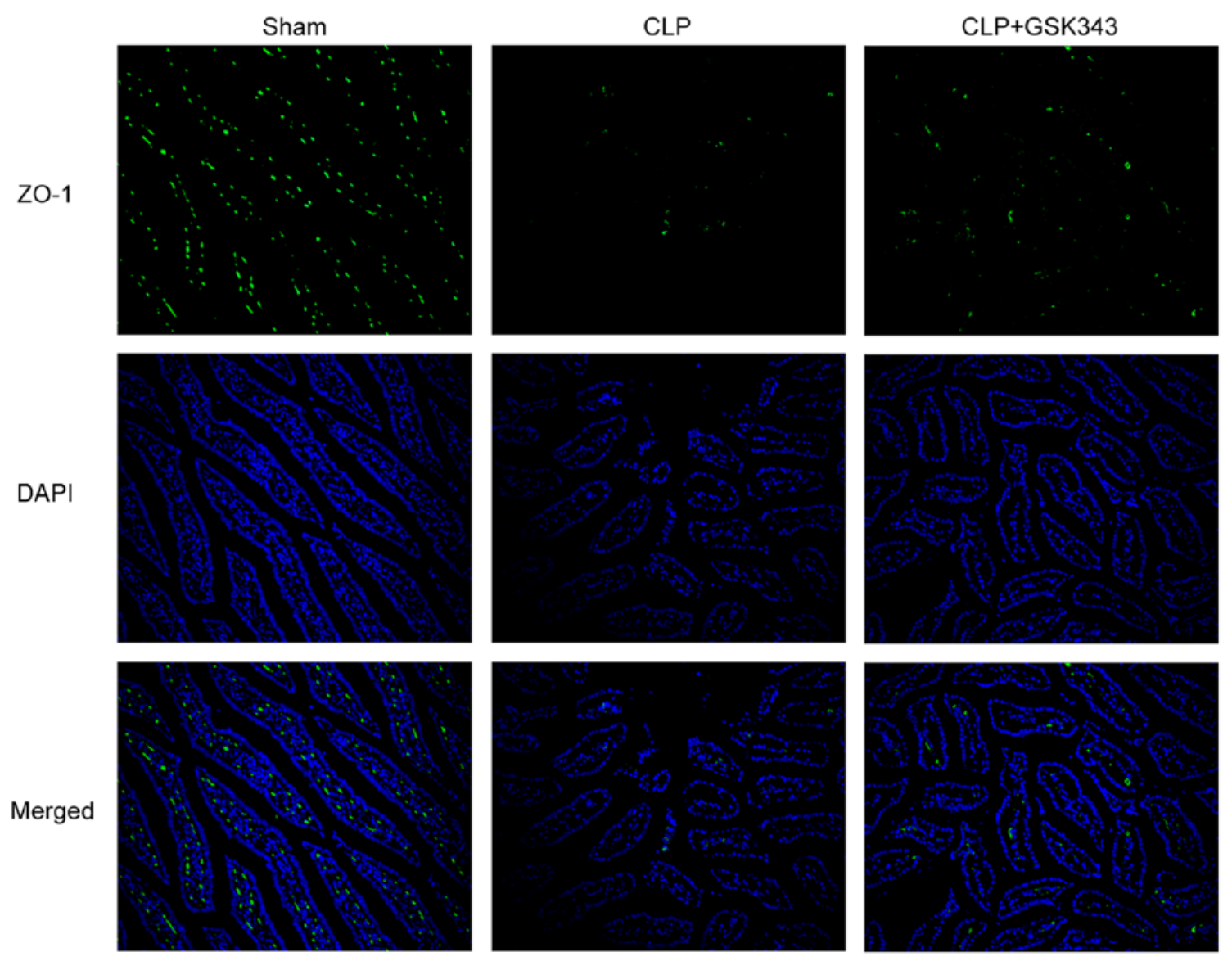

Figure 5. Effects of GSK343 on ZO-1 expression in the intestinal tissues of CLP-induced mice. Images show representative immunofluorescence staining for ZO-1 in the intestinal tissues of mice in the Sham, CLP and CLP + GSK343 group (magnification, x200). CLP, cecal ligation and perforation.

A

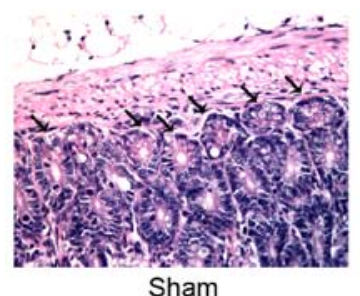

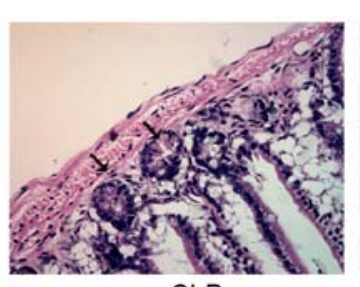

CLP

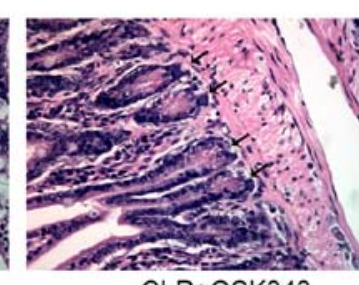

CLP+GSK343
B

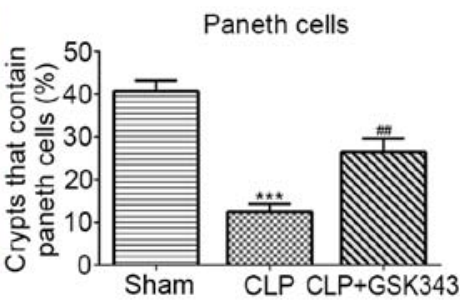

Figure 6. Effects of GSK343 on the distribution of Paneth cells. (A) Representative hematoxylin and eosin staining images for Paneth cells at the base of the intestine crypt based on their distinctive granule staining (arrows; magnification, $\mathrm{x} 400$ ) and (B) quantitative analysis for the number of Paneth cells in the Sham, CLP and CLP + GSK343 group $(\mathrm{n}=10) .{ }^{* * *} \mathrm{P}<0.001$ vs. Sham; ${ }^{* *} \mathrm{P}<0.01$ vs. CLP. CLP, cecal ligation and perforation.

Consequently, the majority of previous studies regarding EZH2 have focused on its modulatory effects on a variety of cancer types, such as prostate cancer (27), ovarian cancer (28), gastric cancer (29) and head and neck cancer (30).

Recently, several studies revealed the involvement of EZH2 in the initiation and progression of sepsis (14,31-33). EZH2 was indicated to accelerate inflammation and apoptosis, and EZH2 inhibition was demonstrated to exert protective effects against sepsis $(14,31,34)$. For example, a study confirmed that GSK343, which is an inhibitor of EZH2, could mitigate fibrosis and inflammation (35). Moreover, EZH2 was indicated to accelerate skeletal muscle cell apoptosis and the inflammatory response in sepsis (31). However, the effects of EZH2 on intestinal dysfunction during sepsis and the underlying epigenetic mechanisms are still unclear. It has been illustrated that epithelial EZH2 was responsible for maintaining the integrity of the epithelial cell barrier and homeostasis, indicating that it has a protective effect on colitis (36). By contrast, the inhibition of EZH2 with GSK126 in vitro has been reported to restore intestinal homeostasis by restoring Paneth cell population, implicating that EZH2 has a destructive role in intestinal homeostasis (37). These seemingly contradictory conclusions reveal the complex and diverse role of EZH2 in intestinal diseases. The present study observed a significant upregulation of EZH2 in CLP-induced septic animal models, suggesting the modulatory effects of EZH2 on intestinal dysfunction during sepsis. Subsequently, septic mice were treated with the EZH2 inhibitor, GSK343. The results indicated that the application 
of GSK343 significantly improved the morphological injury of intestinal tissues, reduced the level of inflammatory cytokines in serum and intestinal tissues, inhibited the occurrence of apoptosis in intestinal tissues and rescued the expression of TJ proteins in intestinal tissues. Furthermore, the decreased population of Paneth cells caused by CLP operation was also significantly increased by GSK343 treatment. These results revealed that intestine injury during sepsis was predominantly caused by inflammation and apoptosis, the relieving of which by EZH2 inhibition was sufficient to prevent the progression of intestinal injury. In addition, the protective effects of EZH2 inhibition against intestine injury during sepsis may mainly rely on suppressing inflammation and apoptosis as well as recovering TJ protein expression and the number of Paneth cells in the intestine. However, there are still some shortcomings in the current study. Firstly, only a CLP-induced mice model was employed in the present study since the laboratory was equipped with mature CLP modeling conditions and technology. Establishment of an LPS-induced mice model should be performed in the future research. The use of clinical samples will strengthen conclusions, and add evidence from clinical samples should be included in future research.

In conclusion, the current study demonstrated that inhibition of EZH2 could relieve intestinal injury during sepsis via suppressing inflammation, apoptosis and at the same time recovering TJ protein expression and the number of Paneth cells in the intestinal tissues. Therefore, targeting EZH2 with pharmacological inhibitors may represent an effective and economical therapeutic approach that may be used in the treatment of intestinal injury caused by sepsis.

\section{Acknowledgements}

Not applicable.

\section{Funding}

No funding was received.

\section{Availability of data and materials}

The datasets used and/or analyzed during the current study are available from the corresponding author on reasonable request.

\section{Authors' contributions}

FW and DY contributed to study conception or design; DY, ZW and YY contributed to acquisition of data; ZH and GL contributed to analysis or interpretation of data; DY and FW drafted the work and revised it critically for important intellectual content. All authors read and approved the final manuscript.

\section{Ethics approval and consent to participate}

The current study was approved by the Ethics Committee of the Second People's Hospital of Zhangye City.

\section{Patient consent for publication}

Not applicable.

\section{Competing interests}

The authors declare that they have no competing interests.

\section{References}

1. Hotchkiss RS, Moldawer LL, Opal SM, Reinhart K, Turnbull IR and Vincent JL: Sepsis and septic shock. Nat Rev Dis Primers 2: 16045, 2016.

2. Fleischmann C, Scherag A, Adhikari NK, Hartog CS, Tsaganos T, Schlattmann P, Angus DC and Reinhart K; International Forum of Acute Care Trialists: Assessment of global incidence and mortality of hospital-treated sepsis. Current estimates and limitations. Am J Respir Crit Care Med 193: 259-272, 2016.

3. Rello J, Valenzuela-Sánchez F, Ruiz-Rodriguez M and Moyano S: Sepsis: A review of advances in management. Adv Ther 34: 2393-2411, 2017.

4. Lelubre $\mathrm{C}$ and Vincent JL: Mechanisms and treatment of organ failure in sepsis. Nat Rev Nephrol 14: 417-427, 2018.

5. Mowat AM and Agace WW: Regional specialization within the intestinal immune system. Nat Rev Immunol 14: 667-685, 2014.

6. Brown EM, Sadarangani M and Finlay BB: The role of the immune system in governing host-microbe interactions in the intestine. Nat Immunol 14: 660-667, 2013.

7. Fay KT, Ford ML and Coopersmith CM: The intestinal microenvironment in sepsis. Biochim Biophys Acta Mol Basis Dis 1863: 2574-2583, 2017.

8. Hwang JS, Kim KH, Park J, Kim SM, Cho H, Lee Y and Han IO: Glucosamine improves survival in a mouse model of sepsis and attenuates sepsis-induced lung injury and inflammation. J Biol Chem 294: 608-622, 2019.

9. Hu Q, Ren H, Li G, Wang D, Zhou Q, Wu J, Zheng J, Huang J, Slade DA, Wu X and Ren J: STING-mediated intestinal barrier dysfunction contributes to lethal sepsis. EBioMedicine 41: 497-508, 2019.

10. Zhou Q and Verne GN: Intestinal hyperpermeability: A gateway to multi-organ failure? J Clin Invest 128: 4764-4766, 2018.

11. Tremblay-LeMay R, Rastgoo N, Pourabdollah M and Chang H: EZH2 as a therapeutic target for multiple myeloma and other haematological malignancies. Biomark Res 6: 34, 2018.

12. Margueron R and Reinberg D: The Polycomb complex PRC2 and its mark in life. Nature 469: 343-349, 2011.

13. Gan L, Yang Y, Li Q, Feng Y, Liu T and Guo W: Epigenetic regulation of cancer progression by EZH2: From biological insights to therapeutic potential. Biomark Res 6: 10, 2018.

14. Zhang Q, Sun H, Zhuang S, Liu N, Bao X, Liu X, Ren H, Lv D, Li Z, Bai J, et al: Novel pharmacological inhibition of EZH2 attenuates septic shock by altering innate inflammatory responses to sepsis. Int Immunopharmacol 76: 105899, 2019.

15. Cazorla SI, Maldonado-Galdeano C, Weill R, De Paula J and Perdigón GD: Oral administration of probiotics increases paneth cells and intestinal antimicrobial activity. Front Microbiol 9: 736, 2018.

16. Livak KJ and Schmittgen TD: Analysis of relative gene expression data using real-time quantitative PCR and the 2(-Delta Delta C(T)) method. Methods 25: 402-408, 2001.

17. Chen S, He Y, Hu Z, Lu S, Yin X, Ma X, Lv C and Jin G: Heparanase mediates intestinal inflammation and injury in a mouse model of sepsis. J Histochem Cytochem 65: 241-249, 2017.

18. Dokladny K, Zuhl MN and Moseley PL: Intestinal epithelial barrier function and tight junction proteins with heat and exercise. J Appl Physiol (1985) 120: 692-701, 2016.

19. Günzel D and Fromm M: Claudins and other tight junction proteins. Compr Physiol 2: 1819-1852, 2012.

20. Zeisel MB, Dhawan P and Baumert TF: Tight junction proteins in gastrointestinal and liver disease. Gut 68: 547-561, 2019.

21. Clevers HC and Bevins CL: Paneth cells: Maestros of the small intestinal crypts. Annu Rev Physiol 75: 289-311, 2013.

22. Schmitt M, Schewe M, Sacchetti A, Feijtel D, van de Geer WS, Teeuwssen M, Sleddens HF, Joosten R, van Royen ME, van de Werken HJ, et al: Paneth cells respond to inflammation and contribute to tissue regeneration by acquiring stem-like features through SCF/c-Kit signaling. Cell Rep 24: 2312-2328.e2317, 2018.

23. Holly MK and Smith JG: Paneth cells during viral infection and pathogenesis. Viruses 10: 225, 2018.

24. Han SJ, Kim M, D'Agati VD and Lee HT: Norepinephrine released by intestinal Paneth cells exacerbates ischemic AKI. Am J Physiol Renal Physiol 318: F260-F272, 2020. 
25. Lee HT, Kim M, Kim JY, Brown KM, Ham A, D'Agati VD and Mori-Akiyama Y: Critical role of interleukin-17A in murine intestinal ischemia-reperfusion injury. Am J Physiol Gastrointest Liver Physiol 304: G12-G25, 2013.

26. Gulati N, Béguelin W and Giulino-Roth L: Enhancer of zeste homolog 2 (EZH2) inhibitors. Leuk Lymphoma 59: 1574-1585, 2018.

27. Bai Y, Zhang Z, Cheng L, Wang R, Chen X, Kong Y, Feng F, Ahmad N, Li L and Liu X: Inhibition of enhancer of zeste homolog 2 (EZH2) overcomes enzalutamide resistance in castration-resistant prostate cancer. J Biol Chem 294: 9911-9923, 2019.

28. Jones BA, Varambally S and Arend RC: Histone methyltransferase EZH2: A therapeutic target for ovarian cancer. Mol Cancer Ther 17: 591-602, 2018

29. Pan YM, Wang CG, Zhu M, Xing R, Cui JT, Li WM, Yu DD Wang SB, Zhu W, Ye YJ, et al: STAT3 signaling drives EZH2 transcriptional activation and mediates poor prognosis in gastric cancer. Mol Cancer 15: 79, 2016.

30. Yamagishi $\mathrm{M}$ and Uchimaru K: Targeting EZH2 in cancer therapy. Curr Opin Oncol 29: 375-381, 2017.

31. Yong H, Wu G, Chen J, Liu X, Bai Y, Tang N, Liu L and Wei J: lncRNA MALAT1 accelerates skeletal muscle cell apoptosis and inflammatory response in sepsis by decreasing BRCA1 expression by recruiting EZH2. Mol Ther Nucleic Acids 19: 97-108, 2020.

32. Zhao D, Li Z, Liu X, Liu N, Bao X, Sun H, Meng Q, Ren H, Bai J, Zhou X and Tang L: Lymphocyte expression of EZH2 is associated with mortality and secondary infectious complications in sepsis. Int Immunopharmacol 89: 107042, 2020.
33. Yong $\mathrm{H}, \mathrm{Wu} \mathrm{G}$, Chen J, Liu X, Bai Y, Tang N, Liu L and Wei J: lncRNA MALAT1 accelerates skeletal muscle cell apoptosis and inflammatory response in sepsis by decreasing BRCA1 expression by recruiting EZH2. Mol Ther Nucleic Acids 21: 1120-1121, 2020.

34. Yu Z, Rayile A, Zhang X, Li Y and Zhao Q: Ulinastatin protects against lipopolysaccharide-induced cardiac microvascular endothelial cell dysfunction via downregulation of lncRNA MALAT1 and EZH2 in sepsis. Int J Mol Med 39: 1269-1276, 2017.

35. Wang Q, Xu L, Zhang X, Liu D and Wang R: GSK343, an inhibitor of EZH2, mitigates fibrosis and inflammation mediated by HIF-1 $\alpha$ in human peritoneal mesothelial cells treated with high glucose. Eur J Pharmacol 880: 173076, 2020.

36. Liu Y, Peng J, Sun T, Li N, Zhang L, Ren J, Yuan H, Kan S, Pan Q, $\mathrm{Li}$ X, et al: Epithelial EZH2 serves as an epigenetic determinant in experimental colitis by inhibiting TNFo-mediated inflammation and apoptosis. Proc Natl Acad Sci USA 114: E3796-E3805, 2017.

37. Nakanishi Y, Reina-Campos M, Nakanishi N, Llado V, Elmen L, Peterson S, Campos A, De SK, Leitges M,Ikeuchi H, et al: Control of paneth cell fate, intestinal inflammation, and tumorigenesis by

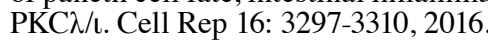

(i)(9) This work is licensed under a Creative Commons Attribution-NonCommercial-NoDerivatives 4.0 International (CC BY-NC-ND 4.0) License. 